\title{
Well-Quasi-Ordering versus Clique-Width: New Results on Bigenic Classes
}

\author{
Konrad K. Dabrowski ${ }^{1}$ Vadim V. Lozin² (D) . \\ Daniël Paulusma ${ }^{1}$
}

Received: 13 November 2016 / Accepted: 13 May 2017 / Published online: 29 June 2017

(C) The Author(s) 2017. This article is an open access publication

\begin{abstract}
Daligault, Rao and Thomassé asked whether a hereditary class of graphs wellquasi-ordered by the induced subgraph relation has bounded clique-width. Lozin, Razgon and Zamaraev recently showed that this is not true for classes defined by infinitely many forbidden induced subgraphs. However, in the case of finitely many forbidden induced subgraphs the question remains open and we conjecture that in this case the answer is positive. The conjecture is known to hold for classes of graphs defined by a single forbidden induced subgraph $H$, as such graphs are well-quasi-ordered and are of bounded clique-width if and only if $H$ is an induced subgraph of $P_{4}$. For bigenic classes of graphs, i.e. ones defined by two forbidden induced subgraphs, there are several open cases in both classifications. In the present paper we obtain a number of new results on well-quasi-orderability of bigenic classes, each of which supports the conjecture.
\end{abstract}

Keywords Well-quasi-order · Induced subgraph $\cdot$ Hereditary graph class $\cdot$ Bigenic class

Research supported by EPSRC (EP/K025090/1 and EP/L020408/1) and the Leverhulme Trust (RPG-2016-258). An extended abstract of this paper appeared in the proceedings of IWOCA 2016 [12].

Konrad K. Dabrowski

konrad.dabrowski@durham.ac.uk

Vadim V. Lozin

v.lozin@warwick.ac.uk

Daniël Paulusma

daniel.paulusma@durham.ac.uk

1 School of Engineering and Computing Sciences, Durham University, Science Laboratories, South Road, Durham DH1 3LE, UK

2 Mathematics Institute, University of Warwick, Coventry CV4 7AL, UK 


\section{Introduction}

Well-quasi-ordering is a highly desirable property and frequently discovered concept in mathematics and theoretical computer science $[18,22]$. One of the most remarkable results in this area is Robertson and Seymour's proof of Wagner's conjecture, which states that the set of all finite graphs is well-quasi-ordered by the minor relation [27]. One of the first steps towards this result was the proof of the fact that graph classes of bounded treewidth are well-quasi-ordered by the minor relation [26] (a graph parameter $\pi$ is said to be bounded for some graph class $\mathcal{G}$ if there exists a constant $c$ such that $\pi(G) \leq c$ for all $G \in \mathcal{G}$ ).

The notion of clique-width generalizes that of treewidth in the sense that graph classes of bounded treewidth have bounded clique-width, but not necessarily vice versa. The importance of both notions is due to the fact that many algorithmic problems that are NP-hard on general graphs become polynomial-time solvable when restricted to graph classes of bounded treewidth or clique-width. For treewidth this follows from the meta-theorem of Courcelle [6], combined with a result of Bodlaender [2]. For clique-width this follows from combining results from several papers $[8,17,20,25]$ with a result of Oum and Seymour [24].

In the study of graph classes of bounded treewidth, we can restrict ourselves to minorclosed graph classes, because from the definition of treewidth it immediately follows that the treewidth of a graph is never smaller than the treewidth of its minor. This restriction, however, is not justified when we study graph classes of bounded clique-width, as the clique-width of a graph can be much smaller than the clique-width of its minor. In particular, Courcelle [7] showed that if $\mathcal{G}$ is the class of graphs of clique-width 3 and $\mathcal{G}^{\prime}$ is the class of graphs obtainable from graphs in $\mathcal{G}$ by applying one or more edge contraction operations, then $\mathcal{G}^{\prime}$ has unbounded clique-width. On the other hand, the clique-width of a graph is never smaller than the clique-width of any of its induced subgraphs (see, for example, [9]). This allows us to restrict ourselves to classes of graphs closed under taking induced subgraphs. Such graph classes are also known as hereditary classes.

It is well-known (and not difficult to see) that a class of graphs is hereditary if and only if it can be characterized by a set of minimal forbidden induced subgraphs. Due to the minimality, the set $\mathcal{F}$ of forbidden induced subgraphs is always an antichain, that is, no graph in $\mathcal{F}$ is an induced subgraph of another graph in $\mathcal{F}$. For some hereditary classes this set is finite, in which case we say that the class is finitely defined, whereas for other hereditary classes (such as, for instance, bipartite graphs) the set of minimal forbidden induced subgraphs forms an infinite antichain. The presence of these infinite antichains immediately shows that the induced subgraph relation is not a well-quasi-order. In fact there even exist graph classes of bounded clique-width that are not well-quasi-ordered by the induced subgraph relation: take, for example, the class of cycles, which all have clique-width at most 4. What about the inverse implication: does well-quasi-ordering imply bounded clique-width? This was stated as an open problem by Daligault, Rao and Thomassé [15] and a negative answer to this question was recently given by Lozin, Razgon and Zamaraev [23]. However, the latter authors disproved the conjecture by giving a hereditary class of graphs whose set of minimal forbidden induced subgraphs is infinite. Hence, for finitely defined classes the question remains open and we conjecture that in this case the answer is positive.

Conjecture 1 If a finitely defined class of graphs $\mathcal{G}$ is well-quasi-ordered by the induced subgraph relation, then $\mathcal{G}$ has bounded clique-width. 
We emphasize that our motivation for verifying Conjecture 1 is not only mathematical but also algorithmic. Should Conjecture 1 be true, then for finitely defined classes of graphs the aforementioned algorithmic consequences of having bounded clique-width also hold for the property of being well-quasi-ordered by the induced subgraph relation.

A class of graphs is monogenic or $\mathrm{H}$-free if it is characterized by a single forbidden induced subgraph $H$. For monogenic classes, the conjecture is true. In this case, the two notions even coincide: a class of graphs defined by a single forbidden induced subgraph $H$ is well-quasi-ordered if and only if it has bounded clique-width if and only if $H$ is an induced subgraph of $P_{4}$ (see, for instance, [14, 16, 21]).

A class of graphs is bigenic or $\left(H_{1}, H_{2}\right)$-free if it is characterized by two incomparable forbidden induced subgraphs $H_{1}$ and $H_{2}$. The family of bigenic classes is more diverse than the family of monogenic classes. The questions of well-quasi-orderability and having bounded clique-width still need to be resolved. Recently, considerable progress has been made towards answering the latter question for bigenic classes; there are currently seven (non-equivalent) open cases [13]. With respect to well-quasi-orderability of bigenic classes, Korpelainen and Lozin [21] left all but 14 cases open. Since then, Atminas and Lozin [1] proved that the class of $\left(K_{3}, P_{6}\right)$-free graphs is well-quasi-ordered by the induced subgraph relation and that the class of $\left(\overline{2 P_{1}+P_{2}}, P_{6}\right)$-free graphs is not. Very recently, we used the techniques developed in the present paper to prove that the classes of $\left(\overline{P_{1}+P_{3}}, P_{2}+P_{4}\right)$ free graphs and $\left(\overline{P_{1}+P_{3}}, P_{1}+P_{5}\right)$-free graphs are also well-quasi-ordered by the induced subgraph relation [13]. The class of $\left(\overline{P_{1}+P_{3}}, P_{1}+2 P_{2}\right)$-free graphs, which is contained in both of these classes, was also an open case. This reduced the number of remaining open cases to nine. All available results for bigenic classes verify Conjecture 1. Moreover, six of the nine open cases have bounded clique-width (and thus immediately verify Conjecture 1), leaving three remaining open cases of bigenic classes for which we still need to verify Conjecture 1.

\subsection{Our Results}

Our first goal is to obtain more (bigenic) classes that are well-quasi-ordered by the induced subgraph relation and to support Conjecture 1 with further evidence. Our second and more general goal is to increase our general knowledge on well-quasi-ordered graph classes and the relation to the possible boundedness of their clique-width.

Towards our first goal we prove in Section 4 that the class of $\left(\overline{2 P_{1}+P_{2}}, P_{2}+P_{3}\right)$-free graphs (which has bounded clique-width [11]) is well-quasi-ordered by the induced subgraph relation. In Section 5 we also determine, by giving infinite antichains, two bigenic classes that are not, namely the class of $\left(\overline{2 P_{1}+P_{2}}, P_{2}+P_{4}\right)$-free graphs, which has unbounded clique-width [11], and the class of $\left(\overline{P_{1}+P_{4}}, P_{1}+2 P_{2}\right)$-free graphs, for which boundedness of the clique-width is unknown (see Fig. 1 for drawings of the five forbidden induced subgraphs). Consequently, there are six classes of $\left(H_{1}, H_{2}\right)$-free graphs for which we do not know whether they are well-quasi-ordered by the induced subgraph relation, and there is one open case left for the verification of Conjecture 1 for bigenic classes. We refer to Open Problems 1 and 3, respectively, in Section 6 where we also give an exact description of the state-of-the-art for results on well-quasi-orderability and boundedness of clique-width for bigenic classes of graphs.

Towards our second goal, we aim to develop general techniques as opposed to tackling specific cases in an ad hoc fashion. Our starting point is a very fruitful technique used for determining (un)boundedness of the clique-width of a graph class $\mathcal{G}$. We transform a given 


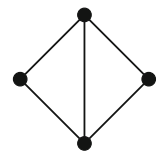

$\overline{2 P_{1}+P_{2}}$

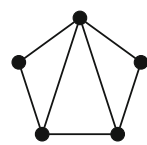

$\overline{P_{1}+P_{4}}$

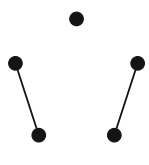

$P_{1}+2 P_{2}$

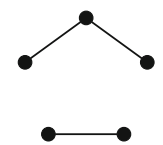

$P_{2}+P_{3}$

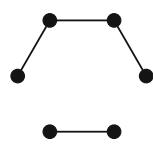

$P_{2}+P_{4}$

Fig. 1 The forbidden induced subgraphs considered in this paper

graph from $\mathcal{G}$ via a number of elementary graph operations that do not modify the cliquewidth by "too much" into a graph from a class for which we do know whether or not its clique-width is bounded.

It is a natural question to research how the above modification technique can be used for well-quasi-orders. We do this in Section 3. The permitted elementary graph operations that preserve (un)boundedness of the clique-width are vertex deletion, subgraph complementation and bipartite complementation. As we will explain in Section 3, these three graph operations do not preserve well-quasi-orderability. We circumvent this by investigating whether these three operations preserve boundedness of a graph parameter called uniformicity. This parameter was introduced by Korpelainen and Lozin [21], who proved that every graph class $\mathcal{G}$ of bounded uniformicity is well-quasi-ordered by the so-called labelled induced subgraph relation, which in turn implies that $\mathcal{G}$ is well-quasi-ordered by the induced subgraph relation. Korpelainen and Lozin [21] proved that boundedness of uniformicity is preserved by vertex deletion. We prove that this also holds for the other two graph operations.

The above enables us to focus on boundedness of uniformicity. However, we cannot always do this: there exist graph classes of unbounded uniformicity that are well-quasiordered by the labelled induced subgraph relation. As such, we sometimes need to rely only on the labelled induced subgraph relation directly. Hence, in Section 3 we also show that the three permitted graph operations, vertex deletion, subgraph complementation and bipartite complementation, preserve well-quasi-orderability by the labelled induced subgraph relation.

As explained in Section 6, we believe that this graph modification technique will also be useful for proving well-quasi-orderability of other graph classes. As such, we view the results in Section 3 as the second main contribution of our paper.

\section{Preliminaries}

The disjoint union $(V(G) \cup V(H), E(G) \cup E(H))$ of two vertex-disjoint graphs $G$ and $H$ is denoted by $G+H$ and the disjoint union of $r$ copies of a graph $G$ is denoted by $r G$. The complement of a graph $G$, denoted by $\bar{G}$, has vertex set $V(\bar{G})=V(G)$ and an edge between two distinct vertices if and only if these vertices are not adjacent in $G$. For a subset $S \subseteq V(G)$, we let $G[S]$ denote the subgraph of $G$ induced by $S$, which has vertex set $S$ and edge set $\{u v \mid u, v \in S, u v \in E(G)\}$. If $S=\left\{s_{1}, \ldots, s_{r}\right\}$ then, to simplify notation, we may also write $G\left[s_{1}, \ldots, s_{r}\right]$ instead of $G\left[\left\{s_{1}, \ldots, s_{r}\right\}\right]$. We use $G \backslash S$ to denote the graph obtained from $G$ by deleting every vertex in $S$, i.e. $G \backslash S=G[V(G) \backslash S]$. We write $H \subseteq \subseteq_{i} G$ to indicate that $H$ is isomorphic to an induced subgraph of $G$.

The graphs $C_{r}, K_{r}, K_{1, r-1}$ and $P_{r}$ denote the cycle, complete graph, star and path on $r$ vertices, respectively. The graph $K_{1,3}$ is also called the claw. The graph $S_{h, i, j}$, for $1 \leq h \leq$ 
$i \leq j$, denotes the subdivided claw, that is, the tree that has only one vertex $x$ of degree 3 and exactly three leaves, which are of distance $h, i$ and $j$ from $x$, respectively. Observe that $S_{1,1,1}=K_{1,3}$. We let $\mathcal{S}$ denote the class of graphs, each connected component of which is either a subdivided claw or a path. For a set of graphs $\left\{H_{1}, \ldots, H_{p}\right\}$, a graph $G$ is $\left(H_{1}, \ldots, H_{p}\right)$-free if it has no induced subgraph isomorphic to a graph in $\left\{H_{1}, \ldots, H_{p}\right\}$; if $p=1$, we may write $H_{1}$-free instead of $\left(H_{1}\right)$-free.

For a graph $G=(V, E)$ and a vertex $u \in V$, the set $N_{G}(u)=\{v \in V \mid u v \in E\}$ denotes the (open) neighbourhood of $u$ in $G$ and $N_{G}[u]=N_{G}(u) \cup\{u\}$ denotes the closed neighbourhood of $u$. We may write $N(u)$ and $N[u]$ instead of $N_{G}(u)$ and $N_{G}[u]$ if this is unambiguous. A graph is bipartite if its vertex set can be partitioned into (at most) two independent sets. The biclique $K_{r, s}$ is the bipartite graph with sets in the partition of size $r$ and $s$ respectively, such that every vertex in one set is adjacent to every vertex in the other set.

Let $X$ be a set of vertices of a graph $G=(V, E)$. A vertex $y \in V \backslash X$ is complete to $X$ if it is adjacent to every vertex of $X$ and anti-complete to $X$ if it is non-adjacent to every vertex of $X$. Similarly, a set of vertices $Y \subseteq V \backslash X$ is complete (resp. anti-complete) to $X$ if every vertex in $Y$ is complete (resp. anti-complete) to $X$. A vertex $y \in V \backslash X$ distinguishes $X$ if $y$ has both a neighbour and a non-neighbour in $X$. The set $X$ is a module of $G$ if no vertex in $V \backslash X$ distinguishes $X$. A module $X$ is non-trivial if $1<|X|<|V|$, otherwise it is trivial. A graph is prime if it has only trivial modules.

A quasi order $\leq$ on a set $X$ is a reflexive, transitive binary relation. Two elements $x, y \in$ $X$ in this quasi-order are comparable if $x \leq y$ or $y \leq x$, otherwise they are incomparable. A set of elements in a quasi-order is a chain if every pair of elements is comparable and it is an antichain if every pair of elements is incomparable. The quasi-order $\leq$ is a well-quasiorder if any infinite sequence of elements $x_{1}, x_{2}, x_{3}, \ldots$ in $X$ contains a pair $\left(x_{i}, x_{j}\right)$ with $x_{i} \leq x_{j}$ and $i<j$. Equivalently, a quasi-order is a well-quasi-order if and only if it has no infinite strictly decreasing sequence $x_{1} \geqslant x_{2} \geqslant x_{3} \geqslant \cdots$ and no infinite antichain.

For an arbitrary set $M$, let $M^{*}$ denote the set of finite sequences of elements of $M$. Any quasi-order $\leq$ on $M$ defines a quasi-order $\leq^{*}$ on $M^{*}$ as follows: $\left(a_{1}, \ldots, a_{m}\right) \leq^{*}$ $\left(b_{1}, \ldots, b_{n}\right)$ if and only if there is a sequence of integers $i_{1}, \ldots, i_{m}$ with $1 \leq i_{1}<\cdots<$ $i_{m} \leq n$ such that $a_{j} \leq b_{i_{j}}$ for $j \in\{1, \ldots, m\}$. We call $\leq^{*}$ the subsequence relation.

Lemma 1 (Higman's Lemma [19]) If $(M, \leq)$ is a well-quasi-order then $\left(M^{*}, \leq^{*}\right)$ is a well-quasi-order.

\subsection{Labelled Induced Subgraphs and Uniformicity}

To define the notion of labelled induced subgraphs, let us consider an arbitrary quasi-order $(W, \leq)$. We say that $G$ is a labelled graph if each vertex $v$ of $G$ is equipped with an element $l_{G}(v) \in W$ (the label of $v$ ). Given two labelled graphs $G$ and $H$, we say that $G$ is a labelled induced subgraph of $H$ if $G$ is isomorphic to an induced subgraph of $H$ and there is an isomorphism that maps each vertex $v$ of $G$ to a vertex $w$ of $H$ with $l_{G}(v) \leq l_{H}(w)$. Clearly, if $(W, \leq)$ is a well-quasi-order, then a class of graphs $X$ cannot contain an infinite sequence of labelled graphs that is strictly-decreasing with respect to the labelled induced subgraph relation. We therefore say that a class of graphs $X$ is well-quasi-ordered by the labelled induced subgraph relation if it contains no infinite antichains of labelled graphs whenever $(W, \leq)$ is a well-quasi-order. Such a class is readily seen to also be well-quasi-ordered by the induced subgraph relation.

We will use the following three results. 
Lemma 2 ([1]) The class of $P_{6}$-free bipartite graphs is well-quasi-ordered by the labelled induced subgraph relation.

Lemma 3 ([1]) Let $k, \ell, m$ be positive integers. Then the class of $\left(P_{k}, K_{\ell}, K_{m, m}\right)$-free graphs is well-quasi-ordered by the labelled induced subgraph relation.

Lemma 4 ([1]) Let $X$ be a hereditary class of graphs. Then $X$ is well-quasi-ordered by the labelled induced subgraph relation if and only if the set of prime graphs in $X$ is. In particular, $X$ is well-quasi-ordered by the labelled induced subgraph relation if and only if the set of connected graphs in $X$ is.

Let $k$ be a natural number, let $K$ be a symmetric square $(0,1)$-matrix of order $k$, and let $F_{k}$ be a graph on the vertex set $\{1,2, \ldots, k\}$. Let $H$ be the disjoint union of infinitely many copies of $F_{k}$, and for $i=1, \ldots, k$, let $V_{i}$ be the subset of $V(H)$ containing vertex $i$ from each copy of $F_{k}$. Now we construct from $H$ an infinite graph $H(K)$ on the same vertex set by applying a subgraph complementation to $V_{i}$ if and only if $K(i, i)=1$ and by applying bipartite complementation to a pair $V_{i}, V_{j}$ if and only if $K(i, j)=1$. In other words, two vertices $u \in V_{i}$ and $v \in V_{j}$ are adjacent in $H(K)$ if and only if $u v \in E(H)$ and $K(i, j)=0$ or $u v \notin E(H)$ and $K(i, j)=1$. Finally, let $\mathcal{P}\left(K, F_{k}\right)$ be the hereditary class consisting of all the finite induced subgraphs of $H(K)$.

Let $k$ be a natural number. A graph $G$ is $k$-uniform if there is a matrix $K$ and a graph $F_{k}$ such that $G \in \mathcal{P}\left(K, F_{k}\right)$. The minimum $k$ such that $G$ is $k$-uniform is the uniformicity of $G$.

The following result was proved by Korpelainen and Lozin. The class of disjoint unions of cliques is a counterexample for the reverse implication.

Theorem 1 ([21]) Any class of graphs of bounded uniformicity is well-quasi-ordered by the labelled induced subgraph relation.

\section{Permitted Graph Operations}

It is not difficult to see that if $G$ is an induced subgraph of $H$, then $\bar{G}$ is an induced subgraph of $\bar{H}$. Therefore, a graph class $X$ is well-quasi-ordered by the induced subgraph relation if and only if the set of complements of graphs in $X$ is. In this section, we strengthen this observation in several ways.

First, we define the operation of subgraph complementation as follows.

Definition 1 Subgraph complementation in a graph $G$ is the operation of complementing a subgraph of $G$ induced by a subset of its vertices.

Applied to the entire vertex set of $G$, this operation coincides with the usual complementation of $G$. However, applied to a pair of vertices, it changes the adjacency of these vertices only. Clearly, repeated applications of this operation can transform $G$ into any other graph on the same vertex set. Therefore, unrestricted applications of subgraph complementation may transform a well-quasi-ordered class $X$ into a class containing infinite antichains. However, if we bound the number of applications of this operation by a constant, we preserve many nice properties of $X$, including well-quasi-orderability with respect to the labelled induced subgraph relation.

Next, we introduce the following operation: 
Definition 2 Bipartite complementation in a graph $G$ is the operation of complementing the edges between two disjoint subsets $X, Y \subseteq V(G)$.

Note that applying a bipartite complementation between $X$ and $Y$ has the same effect as applying a sequence of three subgraph complementations: with respect to $X, Y$ and $X \cup Y$.

Finally, we define the following operation:

Definition 3 Vertex deletion in a graph $G$ is the operation of removing a single vertex $v$ from a graph, together with any edges incident to $v$.

\subsection{Operations on Labelled Graphs}

Let $k \geq 0$ be a constant and let $\gamma$ be a graph operation. A graph class $\mathcal{G}^{\prime}$ is $(k, \gamma)$-obtained from a graph class $\mathcal{G}$ if the following two conditions hold:

(i) every graph in $\mathcal{G}^{\prime}$ is obtained from a graph in $\mathcal{G}$ by performing $\gamma$ at most $k$ times, and

(ii) for every $G \in \mathcal{G}$ there exists at least one graph in $\mathcal{G}^{\prime}$ obtained from $G$ by performing $\gamma$ at most $k$ times.

We say that $\gamma$ preserves well-quasi-orderability by the labelled induced subgraph relation if for any finite constant $k$ and any graph class $\mathcal{G}$, any graph class $\mathcal{G}^{\prime}$ that is $(k, \gamma)$-obtained from $\mathcal{G}$ is well-quasi-ordered by this relation if and only if $\mathcal{G}$ is.

Lemma 5 The following operations preserve well-quasi-orderability by the labelled induced subgraph relation:

(i) Subgraph complementation,

(ii) Bipartite complementation and

(iii) Vertex deletion.

Proof We start by proving the lemma for subgraph complementations.

Let $X$ be a class of graphs and let $Y$ be a set of graphs obtained from $X$ by applying a subgraph complementation to each graph in $X$. More precisely, for each graph $G \in X$ we choose a set $Z_{G}$ of vertices in $G$; we let $G^{\prime}$ be the graph obtained from $G$ by applying a complementation with respect to the subgraph induced by $Z_{G}$ and we let $Y$ be the set of graphs $G^{\prime}$ obtained in this way. Clearly it is sufficient to show that $X$ is well-quasi-ordered by the labelled induced subgraph relation if and only if $Y$ is.

Suppose that $X$ is not well-quasi-ordered under the labelled induced subgraph relation. Then there must be a well-quasi-order $(L, \leq)$ and an infinite sequence $G_{1}, G_{2}, \ldots$ of graphs in $\mathcal{X}$ with vertices labelled with elements of $L$, such that these graphs form an infinite antichain under the labelled induced subgraph relation. Let $\left(L^{\prime}, \leq^{\prime}\right)$ be the quasi-order with $L^{\prime}=\{(k, l): k \in\{0,1\}, l \in L\}$ and $(k, l) \leq^{\prime}\left(k^{\prime}, l^{\prime}\right)$ if and only if $k=k^{\prime}$ and $l \leq l^{\prime}$ (so $L^{\prime}$ is the disjoint union of two copies of $L$, where elements of one copy are incomparable with elements of the other copy). Note that $\left(L^{\prime}, S^{\prime}\right)$ is a well-quasi-order since $(L, \leq)$ is a well-quasi-order.

For each graph $G_{i}$ in this sequence, with labelling $l_{i}$, we construct the graph $G_{i}^{\prime}$ (recall that $G_{i}^{\prime}$ is obtained from $G_{i}$ by applying a complementation on the vertex set $Z_{G_{i}}$ ). We label the vertices of $V\left(G_{i}^{\prime}\right)$ with a labelling $l_{i}^{\prime}$ as follows:

- $\quad$ set $l_{i}^{\prime}(v)=\left(1, l_{i}(v)\right)$ if $v \in Z_{G_{i}}$ and

- $\quad$ set $l_{i}^{\prime}(v)=\left(0, l_{i}(v)\right)$ otherwise. 
We claim that when $G_{1}^{\prime}, G_{2}^{\prime}, \ldots$ are labelled in this way they form an infinite antichain with respect to the labelled induced subgraph relation. Indeed, suppose for contradiction that $G_{i}^{\prime}$ is a labelled induced subgraph of $G_{j}^{\prime}$ for some $i \neq j$. This means that there is a injective map $f: V\left(G_{i}^{\prime}\right) \rightarrow V\left(G_{j}^{\prime}\right)$ such that $l_{i}^{\prime}(v) \leq^{\prime} l_{j}^{\prime}(f(v))$ for all $v \in V\left(G_{i}^{\prime}\right)$ and $v, w \in V\left(G_{i}^{\prime}\right)$ are adjacent in $G_{i}^{\prime}$ if and only if $f(v)$ and $f(w)$ are adjacent in $G_{j}^{\prime}$. Now since $l_{i}^{\prime}(v) \leq^{\prime} l_{j}^{\prime}(f(v))$ for all $v \in V\left(G_{i}^{\prime}\right)$, by the definition of $\leq^{\prime}$ we conclude the following:

- $\quad l_{i}(v) \leq l_{j}(f(v))$ for all $v \in V\left(G_{i}^{\prime}\right)$ and

- $\quad v \in Z_{G_{i}}$ if and only if $f(v) \in Z_{G_{j}}$.

Suppose $v, w \in V\left(G_{i}\right)$ with $w \notin Z_{G_{i}}$ ( $v$ may or may not belong to $Z_{G_{i}}$ ) and note that this implies $f(w) \notin Z_{G_{j}}$. Then $v$ and $w$ are adjacent in $G_{i}$ if and only if $v$ and $w$ are adjacent in $G_{i}^{\prime}$ if and only if $f(v)$ and $f(w)$ are adjacent in $G_{j}^{\prime}$ if and only if $f(v)$ and $f(w)$ are adjacent in $G_{j}$.

Next suppose $v, w \in Z_{G_{i}}$, in which case $f(v), f(w) \in Z_{G_{j}}$. Then $v$ and $w$ are adjacent in $G_{i}$ if and only if $v$ and $w$ are non-adjacent in $G_{i}^{\prime}$ if and only if $f(v)$ and $f(w)$ are non-adjacent in $G_{j}^{\prime}$ if and only if $f(v)$ and $f(w)$ are adjacent in $G_{j}$.

It follows that $f$ is an injective map $f: V\left(G_{i}\right) \rightarrow V\left(G_{j}\right)$ such that $l_{i}(v) \leq l_{j}(f(v))$ for all $v \in V\left(G_{i}\right)$ and $v, w \in V\left(G_{i}\right)$ are adjacent in $G_{i}$ if and only if $f(v)$ and $f(w)$ are adjacent in $G_{j}$. In other words $G_{i}$ is a labelled induced subgraph of $G_{j}$. This contradiction means that if $G_{1}, G_{2}, \ldots$ is an infinite antichain then $G_{1}^{\prime}, G_{2}^{\prime}, \ldots$ must also be an infinite antichain.

Therefore, if the class $X$ is not well-quasi-ordered by the labelled induced subgraph relation then neither is $Y$. Repeating the argument with the roles of $G_{1}, G_{2}, \ldots$ and $G_{1}^{\prime}, G_{2}^{\prime}, \ldots$ reversed shows that if $Y$ is not well-quasi-ordered under the labelled induced subgraph relation then neither is $X$. This completes the proof for subgraph complementations.

Since a bipartite complementation is equivalent to doing three subgraph complementations one after another, the result for bipartite complementations follows.

Finally, we prove the result for vertex deletions.

Let $X$ be a class of graphs and let $Y$ be a set of graphs obtained from $X$ by deleting exactly one vertex $z_{G}$ from each graph $G$ in $X$. We denote the obtained graph by $G-z_{G}$. Clearly it is sufficient to show that $X$ is well-quasi-ordered by the labelled induced subgraph relation if and only if $Y$ is.

Suppose that $Y$ is well-quasi-ordered by the labelled induced subgraph relation. We will show that $X$ is also well-quasi-ordered by this relation. For each graph $G \in X$, let $G^{\prime}$ be the graph obtained from $G$ by applying a bipartite complementation between $\left\{z_{G}\right\}$ and $N\left(z_{G}\right)$, so $z_{G}$ is an isolated vertex in $G^{\prime}$. Let $Z$ be the set of graphs obtained in this way. By Lemma 5.(ii), $Z$ is well-quasi-ordered by the labelled induced subgraph relation if and only if $X$ is. Suppose $G_{1}, G_{2}$ are graphs in $Z$ with vertices labelled from some well-quasiorder $(L, \leq)$. Then for $i \in\{1,2\}$ the vertex $z_{G_{i}}$ has a label from $L$ and the graph $G_{i}-z_{G_{i}}$ belongs to $Y$. Furthermore if $G_{1}-z_{G_{1}}$ is a labelled induced subgraph of $G_{2}-z_{G_{2}}$ and $l_{G_{1}}\left(z_{G_{1}}\right) \leq l_{G_{2}}\left(z_{G_{2}}\right)$ then $G_{1}$ is a labelled induced subgraph of $G_{2}$. Now by Lemma 1 it follows that $Z$ is well-quasi-ordered by the labelled induced subgraph relation. Therefore $X$ is also well-quasi-ordered by this relation.

Now suppose that $Y$ is not well-quasi-ordered by the labelled induced subgraph relation. Then $Y$ contains an infinite antichain $G_{1}, G_{2}, \ldots$ with the vertices of $G_{i}$ labelled by functions $l_{i}$ which takes values in some well-quasi-order $(L, \leq)$. For each $G_{i}$, let $G_{i}^{\prime}$ be a corresponding graph in $X$, so $G_{i}=G_{i}^{\prime}-z_{G_{i}^{\prime}}$. Then in $G_{i}^{\prime}$ we label $z_{G_{i}^{\prime}}$ with a new label * 
and label all other vertices $v \in V\left(G_{i}^{\prime}\right)$ with the same label as that used in $G_{i}$. We make this new label $*$ incomparable to all the other labels in $L$ and note that the obtained quasi order $(L \cup\{*\}, \leq)$ is also a well-quasi-order. It follows that $G_{1}^{\prime}, G_{2}^{\prime}, \ldots$ is an antichain in $X$ when labelled in this way. Therefore, if $Y$ is not well-quasi-ordered by the labelled induced subgraph relation then neither is $X$. This completes the proof.

Note that the above lemmas only apply to well-quasi-ordering with respect to the labelled induced subgraph relation. Indeed, if we take a cycle and delete a vertex, complement the subgraph induced by an edge or apply a bipartite complementation to two adjacent vertices, we obtain a path. However, while the set of cycles is an infinite antichain with respect to the induced subgraph relation, the set of paths is not.

\subsection{Operations on $k$-Uniform Graphs}

We now show that our graph operations do not change uniformicity by "too much" either. The result for vertex deletion was proved by Korpelainen and Lozin.

Lemma 6 Let $G$ be a graph of uniformicity $k$. Let $G^{\prime}, G^{\prime \prime}$ and $G^{\prime \prime \prime}$ be graphs obtained from $G$ by applying one vertex deletion, subgraph complementation or bipartite complementation, respectively. Let $\ell^{\prime}, \ell^{\prime \prime}$ and $\ell^{\prime \prime \prime}$ be the uniformicities of $G^{\prime}, G^{\prime \prime}$ and $G^{\prime \prime \prime}$, respectively. Then the following three statements hold:

(i) $\ell^{\prime}<k<2 \ell^{\prime}+1[21]$;

(ii) $\frac{k}{2} \leq \ell^{\prime \prime} \leq 2 k$;

(iii) $\frac{k}{3} \leq \ell^{\prime \prime \prime} \leq 3 k$.

Proof The first inequality of Part (i) is trivial. The second inequality of Part (i) was proved in [21]. We prove Parts (ii) and (iii) below.

Let $G$ be a graph of uniformicity $k$, let $X$ be a set of vertices in $G$ and let $G^{\prime \prime}$ be the graph obtained from $G$ by applying a complementation with respect to the subgraph induced by $X$. Let $\ell^{\prime \prime}$ be the uniformicity of $G^{\prime \prime}$. By symmetry, to prove Part (ii), it is sufficient to prove that $\ell^{\prime \prime} \leq 2 k$.

Since $G$ is a $k$-uniform graph, it must belong to $P\left(F_{k}, K\right)$ for some $F_{k}$ and some $K$, so it is an induced subgraph of $H(K)$.

Consider the graph obtained from $F_{k}$ by replacing each vertex $v$ of $F_{k}$ by two nonadjacent vertices $v$ and $v^{\prime}$. Apply a complementation with respect to $\left\{1^{\prime}, 2^{\prime}, \ldots, k^{\prime}\right\}$ and let $F_{k}^{\prime}$ be the obtained graph. In other words, if $v \in V\left(F_{k}\right)$ then

$$
\begin{aligned}
N_{F_{k}^{\prime}}(v) & =N_{F_{k}}(v) \cup\left\{w^{\prime} \mid w \in N_{F_{k}}(v)\right\} \text { and } \\
N_{F_{k}^{\prime}}\left(v^{\prime}\right) & =N_{F_{k}}(v) \cup\left\{w^{\prime} \mid w \in V\left(F_{k}\right) \backslash N_{F_{k}}[v]\right\} .
\end{aligned}
$$

Let $K^{\prime}$ be a $2 k \times 2 k$ matrix indexed by $\left\{1,2, \ldots, k, 1^{\prime}, 2^{\prime}, \ldots, k^{\prime}\right\}$ with $K_{i, j}^{\prime}=K_{i, j^{\prime}}^{\prime}=$ $K_{i^{\prime}, j}^{\prime}=1-K_{i^{\prime}, j^{\prime}}^{\prime}=K_{i, j}$.

This means that $H\left(K^{\prime}\right)$ is formed from $H(K)$ by adding a copy of each vertex that has the same neighbourhood and then applying a complementation with respect to the set of newly-created vertices.

Similarly, $G^{\prime \prime}$ can be obtained from $G$ by replacing each vertex in $X$ by a copy with the same neighbourhood and then applying a complementation with respect to the set of 
newly-created vertices. Therefore $G^{\prime \prime}$ is an induced subgraph of $H\left(K^{\prime}\right)$. Therefore $G^{\prime \prime}$ is a $2 k$-uniform graph.

Part (iii) follows from similar arguments to those for Part (ii). (Also note that if we weaken the bounds in Part (iii) to $\frac{k}{8} \leq \ell^{\prime \prime \prime} \leq 8 k$ then the result follows immediately from combining Part (ii) with the fact that a bipartite complementation is equivalent to a sequence of three subgraph complementations).

\section{A New Well-Quasi-Ordered Class}

In this section we show that $\left(\overline{2 P_{1}+P_{2}}, P_{2}+P_{3}\right)$-free graphs are well-quasi-ordered by the labelled induced subgraph relation. We divide the proof into several sections, depending on whether or not the graphs under consideration contain certain induced subgraphs or not. We follow the general scheme that Dabrowski, Huang and Paulusma [11] used to prove that this class has bounded clique-width, but we will also need a number of new arguments.

\subsection{Graphs containing a $K_{5}$}

We first consider graphs containing a $K_{5}$ and prove the following lemma.

Lemma 7 The class of $\left(\overline{2 P_{1}+P_{2}}, P_{2}+P_{3}\right)$-free graphs that contain a $K_{5}$ is well-quasiordered by the labelled induced subgraph relation.

Proof Let $G$ be a $\left(\overline{2 P_{1}+P_{2}}, P_{2}+P_{3}\right)$-free graph. Let $X$ be a maximal (by set inclusion) clique in $G$ containing at least five vertices.

Claim 1. Every vertex not in $X$ has at most one neighbour in $X$.

This follows from the fact that $X$ is maximal and $G$ is $\overline{2 P_{1}+P_{2}}$-free.

Suppose there is a $P_{3}$ in $G \backslash X$, say on vertices $x_{1}, x_{2}, x_{3}$ in that order. Since $|X| \geq$ 5 , we can find $y_{1}, y_{2} \in X$ none of which are adjacent to any of $x_{1}, x_{2}, x_{3}$. Then $G\left[y_{1}, y_{2}, x_{1}, x_{2}, x_{3}\right]$ is a $P_{2}+P_{3}$. This contradiction shows that $G \backslash X$ is $P_{3}$-free and must therefore be a union of disjoint cliques $X_{1}, \ldots, X_{k}$. We say that a clique $X_{i}$ is large if it contains at least two vertices and that it is small if it contains exactly one vertex.

Claim 2. If $x \in X$ is adjacent to $y \in X_{i}$ and $X_{j}$ (with $i \neq j$ ) is large, then $x$ can have at most one non-neighbour in $X_{j}$.

For contradiction, assume that $x$ is non-adjacent to $z_{1}, z_{2} \in X_{j}$. Since $|X| \geq 5$ and each vertex that is not in $X$ has at most one neighbour in $X$, there must be a vertex $x^{\prime} \in X$ that is non-adjacent to $y, z_{1}$ and $z_{2}$. Then $G\left[z_{1}, z_{2}, x^{\prime}, x, y\right]$ is a $P_{2}+P_{3}$, a contradiction.

We consider several cases:

Case 1: $G \backslash X$ contains at most one clique.

Then the complement of $G$ is a bipartite graph. Moreover, since the complement of $\overline{2 P_{1}+P_{2}}$ is an induced subgraph of $P_{6}$, we conclude that the complements of graphs in our class form a subclass of $P_{6}$-free bipartite graphs, which are well-quasi-ordered by the labelled induced subgraph relation by Lemmas 2 and 5.(i). 
Case 2: $G \backslash X$ does not contain large cliques.

In this case, the structure of graphs can be described as follows: take a collection of stars and create a clique on their central vertices and then add a number (possibly zero) of isolated vertices. In other words, applying subgraph complementation once (to the clique $X$ ), we obtain a graph which is a disjoint union of stars and isolated vertices. Clearly, a graph every connected component of which is a star or an isolated vertex is $P_{6}$-free bipartite and hence by Lemmas 2 and 5.(i) we conclude that graphs in our class are well-quasi-ordered by the labelled induced subgraph relation.

Case 3: $G \backslash X$ contains exactly one large clique.

Without loss of generality, assume that $X_{1}$ is large and the remaining cliques $X_{2}, \ldots, X_{k}$ are small. Suppose there are $\ell$ distinct vertices $x_{1}, \ldots, x_{\ell} \in X$, each of which has a neighbour in $X_{2} \cup \cdots \cup X_{k}$. By Claim 2, each of these vertices has at most one non-neighbour in $X_{1}$. But then $\ell \leq 2$, since otherwise a vertex of $X_{1}$ has more than one neighbour in $X$.

Therefore, by deleting at most two vertices from $G$ we transform it to a graph from Case 1 plus a number of isolated vertices. Lemma 4 allows us to ignore isolated vertices, while Lemma 5.(iii) allows the deletion of finitely many vertices. Therefore, in Case 3 we deal with a set of graphs which is well-quasi-ordered by the labelled induced subgraph relation.

Case 4: $G \backslash X$ contains at least two cliques that are large.

Suppose there is a vertex $x \in X$ that has a neighbour outside of $X$. By Claim 2, $x$ has at most one non-neighbour in each large clique. Therefore, at most two vertices of $X$ have neighbours outside of $X$, since otherwise each large clique would have a vertex with more than one neighbour in $X$. But then by deleting at most two vertices we transform $G$ into a $P_{3}$-free graph (i.e. a graph every connected component of which is a clique). It is wellknown (and also follows from Lemma 4 ) that the set of $P_{3}$-free graphs is well-quasi-ordered by the labelled induced subgraph relation. Therefore, by Lemma 5.(iii), the same is true for graphs in Case 4.

\subsection{Graphs containing a $C_{5}$}

By Lemma 7, we may restrict ourselves to looking at $K_{5}$-free graphs in our class. We now consider the case where these graphs have an induced $C_{5}$.

Lemma 8 The class of $\left.\overline{\left(2 P_{1}+P_{2}\right.}, P_{2}+P_{3}, K_{5}\right)$-free graphs that contain an induced $C_{5}$ has bounded uniformicity.

Proof To prove this, we modify the proof from [11], which shows that this class of graphs has bounded clique-width. Let $G$ be a $\left(\overline{2 P_{1}+P_{2}}, P_{2}+P_{3}, K_{5}\right)$-free graph containing a $C_{5}$, say on vertices $v_{1}, v_{2}, v_{3}, v_{4}, v_{5}$ in order. Our goal is to show that the graph $G$ has bounded uniformicity and hence, by Lemma 6.(i), in the proof we can neglect any set of vertices that is bounded in size by a constant.

Let $Y$ be the set of vertices adjacent to $v_{1}$ and $v_{2}$ (and possibly to other vertices on the cycle). If $y_{1}, y_{2} \in Y$ are non-adjacent, then $G\left[v_{1}, v_{2}, y_{1}, y_{2}\right]$ would be a $\overline{2 P_{1}+P_{2}}$. Therefore, $Y$ is a clique. This clique has at most two vertices, since otherwise three vertices of $Y$ together with $v_{1}$ and $v_{2}$ would create a $K_{5}$. Therefore, the set of all vertices with two consecutive neighbours on the cycle is finite and hence can be neglected (removed from the graph). We may therefore assume that each vertex not on the cycle has at most two neighbours on the cycle and if it has two such neighbours, they must be non-consecutive vertices of the cycle. 
Now let $W$ be the set of vertices whose unique neighbour on the cycle is $v_{1}$. If $y_{1}, y_{2} \in W$ are non-adjacent, then $G\left[v_{3}, v_{4}, y_{1}, v_{1}, y_{2}\right]$ would be a $P_{2}+P_{3}$. If $y_{1}, y_{2} \in W$ are adjacent, then $G\left[y_{1}, y_{2}, v_{2}, v_{3}, v_{4}\right]$ would be a $P_{2}+P_{3}$. Therefore, $W$ contains at most one vertex, and hence the set of all vertices with exactly one neighbour on the cycle can be removed from the graph.

Let $X$ be the set of vertices with no neighbours on the cycle. $X$ must be an independent set, since if two vertices in $x_{1}, x_{2} \in X$ are adjacent, then $G\left[x_{1}, x_{2}, v_{1}, v_{2}, v_{3}\right]$ would induce a $P_{2}+P_{3}$ in $G$.

For $i \in\{1,2,3,4,5\}$, let $V_{i}$ be the set of vertices not on the cycle that are adjacent to $v_{i-1}$ and $v_{i+1}$, but non-adjacent to all other vertices of the cycle (subscripts on vertices and vertex sets are interpreted modulo 5 throughout this proof). For each $i$, the set $V_{i}$ must be independent, since if $x, y \in V_{i}$ are adjacent then $G\left[x, y, v_{i-1}, v_{i+1}\right]$ is a $\overline{2 P_{1}+P_{2}}$.

We say that two sets $V_{i}$ and $V_{j}$ are consecutive (respectively opposite) if $v_{i}$ and $v_{j}$ are distinct adjacent (respectively non-adjacent) vertices of the cycle. We say that a set $X$ or $V_{i}$ is large if it contains at least three vertices, otherwise it is small. We say that a bipartite graph with bipartition classes $A$ and $B$ is a matching (co-matching) if every vertex in $A$ has at most one neighbour (non-neighbour) in $B$, and vice versa.

Dabrowski, Huang and Paulusma proved the following claims about the edges between these sets (see Appendix A for a proof).

Claim 1. ([11]) $G\left[V_{i} \cup X\right]$ is a matching.

Claim 2. ([11]) If $V_{i}$ and $V_{j}$ are opposite, then $G\left[V_{i} \cup V_{j}\right]$ is a matching.

Claim 3. ([11]) If $V_{i}$ and $V_{j}$ are consecutive, then $G\left[V_{i} \cup V_{j}\right]$ is a co-matching.

Claim 4. ([11]) If $V_{i}$ is large, then $X$ is anti-complete to $V_{i-2} \cup V_{i+2}$.

Claim 5. ([11]) If $V_{i}$ is large, then $V_{i-1}$ is anti-complete to $V_{i+1}$.

Claim 6. ([11]) If $V_{i-1}, V_{i}, V_{i+1}$ are large, then $V_{i}$ is complete to $V_{i-1} \cup V_{i+1}$.

We also prove the following claim:

Claim 7. Suppose two consecutive sets $V_{i}$ and $V_{i+1}$ are large and a vertex $y \in V_{i}$ is not adjacent to a vertex $z \in V_{i+1}$. Then every vertex $x \in X \cup V_{i+3}$ is either complete or anticomplete to $\{y, z\}$.

To prove this, suppose for contradiction that this is not the case. Without loss of generality, we may assume that that $x$ is adjacent to $y$ but not to $z$. Since $V_{i}$ is large it contains at least two other vertices, say $a$ and $b$. Then $z$ is adjacent to both $a$ and $b$ (since $G\left[V_{i} \cup V_{i+1}\right]$ is a co-matching), while $x$ is adjacent neither to $a$ nor to $b$ (since $G\left[V_{i} \cup X\right]$ and $G\left[V_{i} \cup V_{i+3}\right]$ are matchings). But then $G[x, y, a, z, b]$ is a $P_{2}+P_{3}$, a contradiction.

We are now ready to prove the lemma. We may delete from $G$ the vertices $v_{1}, \ldots, v_{5}$ and all vertices in every small set $X$ or $V_{i}$. Let $G^{\prime}$ be the resulting graph. In order to show that $G^{\prime}$ has bounded uniformicity, we split the analysis into the following cases.

Case 1: All sets $V_{1}, \ldots, V_{5}$ are large.

From the above claims we conclude that any two consecutive sets are complete to each other and any two opposite sets are anti-complete to each other. Also, $X$ is anti-complete to each of them. Therefore $G^{\prime}$ is 6-uniform.

Case 2: Four sets $V_{i}$ are large, say $V_{1}, \ldots, V_{4}$.

Then $V_{1}$ and $V_{4}$ form a matching, while any other pair of these sets are either complete or anti-complete to each other. Also, $X$ is anti-complete to each of them. Therefore $G^{\prime}$ is 5-uniform. 
Case 3: Three consecutive sets $V_{i}$ are large, say $V_{1}, V_{2}, V_{3}$.

Then $V_{2}$ is complete to $V_{1}$ and $V_{3}$, while $V_{1}$ and $V_{3}$ are anti-complete to each other. Also, $X$ is anti-complete to $V_{1}$ and $V_{3}$ forms a matching with $V_{2}$. Therefore $G^{\prime}$ is 4-uniform.

Case 4: Three non-consecutive sets $V_{i}$ are large, say $V_{1}, V_{3}, V_{4}$.

Then $X$ is anti-complete to each of them. From the above claims we know that $V_{1}$ forms a matching with both $V_{3}$ and $V_{4}$, while $V_{3}$ and $V_{4}$ form a co-matching. Also, from Claim 7 we conclude that whenever two vertices $y \in V_{3}$ and $z \in V_{4}$ are non-adjacent, then either none of them has a neighbour in $V_{1}$ or they both are adjacent to the same vertex of $V_{1}$. Therefore, if we complement the edges between $V_{3}$ and $V_{4}$, then $G^{\prime}$ transforms into a graph in which every connected component is one of the following graphs: $K_{3}, P_{3}, P_{2}, P_{1}$. Each of these graphs is an induced subgraph of $\overline{P_{1}+P_{3}}$ (also known as the paw), so the obtained graph is 4-uniform. By Lemma 6.(iii), it follows that $G\left[V_{1} \cup V_{3} \cup V_{4}\right]$ is 12-uniform and so $G^{\prime}$ is 13-uniform.

Case 5: Two consecutive sets $V_{i}$ are large, say $V_{3}, V_{4}$.

This case is similar to the previous one, where the role of $V_{1}$ is played by $X$. Thus $G^{\prime}$ is 12-uniform.

Case 6: Two non-consecutive sets $V_{i}$ are large.

Then $X$ is anti-complete to each of them and hence the graph is obviously 3-uniform.

Case 7: At most one set $V_{i}$ is large.

Then $G^{\prime}$ is obviously 2-uniform.

Since the above cases cover all possibilities, this completes the proof.

\subsection{Graphs Containing a $C_{4}$}

By Lemmas 7 and 8, we may restrict ourselves to looking at $\left(K_{5}, C_{5}\right)$-free graphs in our class. We prove the following structural result.

Lemma 9 Let $G$ be a $\left(\overline{2 P_{1}+P_{2}}, P_{2}+P_{3}, K_{5}, C_{5}\right)$-free graph containing an induced $C_{4}$. Then by deleting at most 17 vertices and applying at most two bipartite complementations, we can modify $G$ into the disjoint union of a $\left(P_{2}+P_{3}\right)$-free bipartite graph and a 3-uniform graph.

Proof In order to prove the lemma, we again modify the proof from [11], which shows that this class of graphs has bounded clique-width. Let $G$ be a $\left.\overline{\left(2 P_{1}+P_{2}\right.}, P_{2}+P_{3}, K_{5}, C_{5}\right)$-free graph containing a $C_{4}$ induced by the vertices $v_{1}, v_{2}, v_{3}, v_{4}$ in order. We interpret subscripts on vertices modulo 4 in this proof.

Let $Y$ be the set of vertices adjacent to $v_{1}$ and $v_{2}$ (and possibly to other vertices on the cycle). If $y_{1}, y_{2} \in Y$ are non-adjacent, then $G\left[v_{1}, v_{2}, y_{1}, y_{2}\right]$ would be a $\overline{2 P_{1}+P_{2}}$. Therefore, $Y$ is a clique. This clique has at most two vertices, since otherwise three vertices of $Y$ together with $v_{1}$ and $v_{2}$ would create a $K_{5}$. Therefore, after deleting at most $2 \times 4=8$ vertices, we may assume that no vertex of the graph contains two consecutive neighbours on the cycle.

Let $V_{1}$ denote the set of vertices adjacent to $v_{2}$ and $v_{4}$, and let $V_{2}$ denote the set of vertices adjacent to $v_{1}$ and $v_{3}$. For $i \in\{1,2,3,4\}$, let $W_{i}$ denote the set of vertices whose only neighbour on the cycle is $v_{i}$. If a set $W_{i}$ contains at most one vertex then we may delete this vertex. Thus, deleting at most four vertices, we may assume that every set $W_{i}$ is either 
empty of contains at least two vertices. Finally, let $X$ be the set of vertices with no neighbour on the cycle.

Dabrowski, Huang and Paulusma proved the following claims about the edges between these sets (see Appendix B for a proof).

Claim 1. ([11]) $V_{i}$ is independent for $i \in\{1,2\}$.

Claim 2. ([11]) $W_{i}$ is independent for $i \in\{1,2,3,4\}$.

Claim 3. ([11]) $X$ is independent.

Claim 4. ([11]) $W_{i}$ is anti-complete to $X$ for $i \in\{1,2,3,4\}$.

Claim 5. ([11]) For $i \in\{1,2\}$ either $W_{i}$ or $W_{i+2}$ is empty. Therefore, we may assume by symmetry that $W_{3}=\emptyset$ and $W_{4}=\emptyset$.

Note that in our arguments so far we have deleted at most 12 vertices. We now argue as follows:

Any vertices of $X$ that do not have neighbours in $V_{1} \cup V_{2}$ must be isolated vertices of the graph. Since adding isolated vertices to a $P_{2}+P_{3}$-free bipartite graph maintains the property of it being $P_{2}+P_{3}$-free and bipartite, we may therefore assume that every vertex in $X$ has a neighbour in $V_{1} \cup V_{2}$. Let $X_{0}$ denote the subset of $X$ whose vertices have neighbours both in $V_{1}$ and $V_{2}$, let $X_{1}$ denote the subset of $X$ whose vertices have no neighbours in $V_{1}$ and let $X_{2}$ denote the subset of $X$ whose vertices have no neighbours in $V_{2}$.

Let $V_{0}$ denote the set of vertices in $V_{1} \cup V_{2}$ adjacent to at least one vertex of $X_{0}$ and let $V_{10}=V_{1} \cap V_{0}$ and $V_{20}=V_{2} \cap V_{0}$. If $V_{10}$ or $V_{20}$ contains at most one vertex then we may delete this vertex. This would cause $X_{0}$ to become empty. Therefore, by deleting at most one vertex, we may assume that either both $V_{10}$ and $V_{20}$ each contain at least two vertices or else $V_{10}, V_{20}$ and $X_{0}$ are all empty. We will show that $G\left[X_{0} \cup V_{0}\right]$ is 3-uniform and can be separated from the rest of the graph using at most two bipartite complementations. To do this, we first prove the following additional claims.

Claim 6. Every vertex of $X_{0}$ has exactly one neighbour in $V_{10}$ and exactly one neighbour in $V_{20}$ and these neighbours are adjacent.

First, we observe that if a vertex $x \in X_{0}$ is adjacent to $y \in V_{1}$ and to $z \in V_{2}$, then $y$ is adjacent to $z$, since otherwise $G\left[x, y, v_{2}, v_{1}, z\right]$ is an induced $C_{5}$. This implies that if $x$ has the third neighbour $y^{\prime} \in V_{1} \cup V_{2}$, then $G\left[x, z, y, y^{\prime}\right]$ is a $\overline{2 P_{1}+P_{2}}$. This contradiction proves the claim.

Claim 7. Every vertex of $V_{0}$ is adjacent to exactly one vertex of $X_{0}$.

Let $v$ be a vertex in $V_{0}$. Without loss of generality, assume that $v$ belongs to $V_{10}$. Suppose that $v$ has at least two neighbours in $X_{0}$, say $x$ and $x^{\prime}$, and at least one non-neighbour, say $x^{\prime \prime}$. Let $v^{\prime \prime}$ be the neighbour of $x^{\prime \prime}$ in $V_{10}$. Then $G\left[v^{\prime \prime}, x^{\prime \prime}, x, v, x^{\prime}\right]$ is a $P_{2}+P_{3}$. This contradiction shows that if $v$ has at least two neighbours in $X_{0}$, then it must be adjacent to all the vertices of $X_{0}$. Since every vertex of $X_{0}$ has exactly one neighbour in $V_{10}$ it follows that $v$ is the only vertex of $V_{10}$, a contradiction. We conclude that $v$ (and hence every other vertex of $V_{0}$ ) has exactly one neighbour in $X_{0}$.

Claim 8. $V_{10}$ is complete to $V_{2}$ and $V_{20}$ is complete to $V_{1}$.

Suppose $v \in V_{10}$ is non-adjacent to $y \in V_{2}$ and let $x$ be the unique neighbour of $v$ in $X_{0}$. Since $y$ is non-adjacent to $v$, it cannot be the unique neighbour of $x$ in $V_{2}$. Therefore $y$ must be non-adjacent to $x$. It follows that $G\left[x, v, v_{1}, y, v_{3}\right]$ is a $P_{2}+P_{3}$, a contradiction. The second part of the claim follows by symmetry. 
Claim 9. Every vertex in $W_{1} \cup W_{2} \cup X_{1} \cup X_{2}$ is either complete or anti-complete to $V_{i 0}$ for $i=1,2$.

Suppose a vertex $w \in W_{1} \cup W_{2} \cup X_{1} \cup X_{2}$ has both a neighbour $v$ and a non-neighbour $v^{\prime}$ in $V_{10}$. Let $x$ and $x^{\prime}$ be the neighbours of $v$ and $v^{\prime}$, respectively, in $X_{0}$. Recall that $x$ and $x^{\prime}$ must be non-adjacent to $w$. Then $G\left[v^{\prime}, x^{\prime}, w, v, x\right]$ is a $P_{2}+P_{3}$, a contradiction.

By Claims 8 and 9, every vertex outside $V_{10} \cup V_{20} \cup X_{0}$ is either complete or anticomplete to $V_{10}$ and either complete or anti-complete to $V_{20}$. Applying at most two bipartite complementations, we may therefore disconnect $G\left[V_{10} \cup V_{20} \cup X_{0}\right]$ from the rest of the graph i.e. remove all edges between vertices in $V_{10} \cup V_{20} \cup X_{0}$ and vertices outside $V_{10} \cup V_{20} \cup X_{0}$. By Claims 6, 7 and 8 it follows that $G\left[V_{10} \cup V_{20} \cup X_{0}\right]$ is a 3-uniform graph.

We may now assume that $X_{0}$ is empty. Let $H$ be the graph obtained from $G$ by deleting the vertices of the original cycle. Note that $V(H)=X_{1} \cup V_{1} \cup W_{1} \cup X_{2} \cup V_{2} \cup W_{2}$. It remains to show that $H$ is a $\left(P_{2}+P_{3}\right)$-free bipartite graph.

We claim that $H$ is bipartite with independent sets $X_{1} \cup V_{1} \cup W_{1}$ and $X_{2} \cup V_{2} \cup W_{2}$. To show this, it suffices to prove that $H$ has no triangles, because all other odd cycles are forbidden in $G$ (and hence in $H$ ). We know that $X_{1} \cup V_{1}$ is an independent set and $X_{2} \cup V_{2}$ is an independent set. Also, $W_{1}$ and $W_{2}$ are independent and the vertices of $X$ have no neighbours in $W_{1} \cup W_{2}$. It follows that in $H$ vertices in $X_{1}$ an can only have neighbours in $V_{2}$ and vertices of $X_{2}$ can only have neighbours in $V_{1}$, so no triangle in $H$ contains a vertex of $X$. By symmetry, if $H[x, y, z]$ is a triangle then we may therefore assume that $x \in W_{1}, y \in V_{2}$ and $z \in V_{1} \cup W_{2}$. Now $G\left[x, y, z, v_{1}\right]$ is a $\overline{2 P_{1}+P_{2}}$, a contradiction. It follows that $H$ is bipartite. Moreover, it is $\left(P_{2}+P_{3}\right)$-free. This completes the proof.

Since $P_{2}+P_{3}$ is an induced subgraph of $P_{6}$, it follows that every $\left(P_{2}+P_{3}\right)$-free graph is $P_{6}$-free. Combining Lemma 9 with Theorem 1 and Lemmas 2, 5.(ii) and 5.(iii) we therefore obtain the following corollary.

Corollary 1 The class of $\left(\overline{2 P_{1}+P_{2}}, P_{2}+P_{3}, K_{5}, C_{5}\right)$-free graphs containing an induced $C_{4}$ is well-quasi-ordered by the labelled induced subgraph relation.

\section{$4.4\left(\overline{2 P_{1}+P_{2}}, P_{2}+P_{3}\right)-$ free graphs}

Theorem 2 The class of $\left(\overline{2 P_{1}+P_{2}}, P_{2}+P_{3}\right)$-free graphs is well-quasi-ordered by the labelled induced subgraph relation.

Proof Graphs in the class under consideration containing an induced subgraph isomorphic to $K_{5}, C_{5}$ or $C_{4}$ are well-quasi-ordered by the labelled induced subgraph relation by Lemmas 7 and 8 and Corollary 1, respectively. The remaining graphs form a subclass of $\left(P_{6}, K_{5}, K_{2,2}\right.$ )-free graphs, since $C_{4}=K_{2,2}$ and $P_{2}+P_{3}$ is an induced subgraph of $P_{6}$. By Lemma 3 , this class of graphs is well-quasi-ordered by the labelled induced subgraph relation. Therefore, the class of $\left(\overline{2 P_{1}+P_{2}}, P_{2}+P_{3}\right)$-free graphs is well-quasi-ordered by the labelled induced subgraph relation.

\section{Two New Non-Well-Quasi-Ordered Classes}

In this section we show that the classes of $\left(\overline{2 P_{1}+P_{2}}, P_{2}+P_{4}\right)$-free graphs and $\left(\overline{P_{1}+P_{4}}, P_{1}+2 P_{2}\right)$-free graphs are not well-quasi-ordered by the induced subgraph relation. The antichain used to prove the first of these cases was previously used by Atminas 


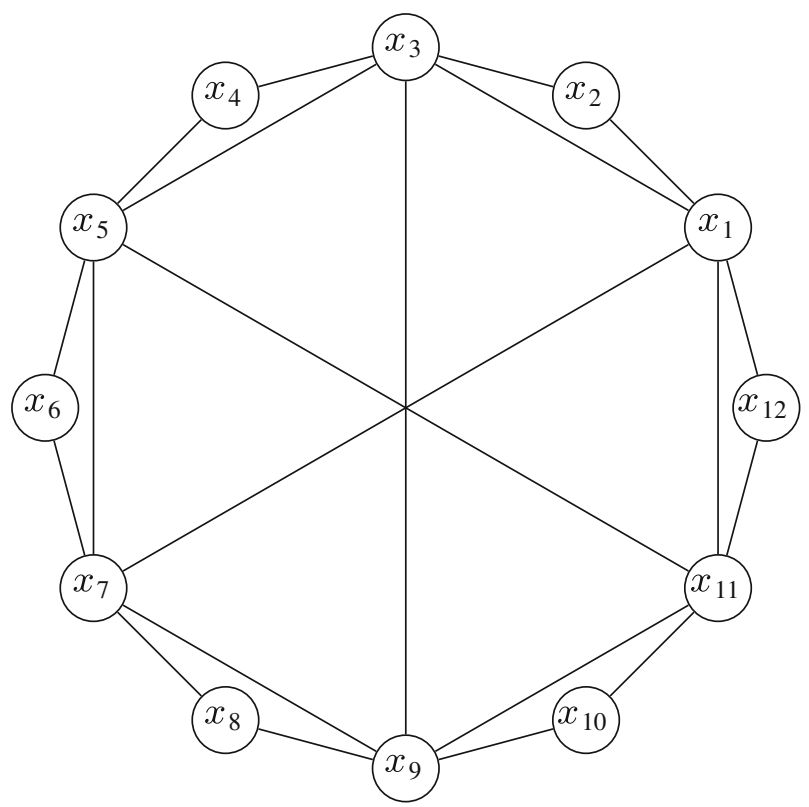

Fig. 2 The Graph $G_{4 n}$ from Theorem 3 when $n=3$

and Lozin to show that the class of $\left.\overline{\left(2 P_{1}+P_{2}\right.}, P_{6}\right)$-free graphs is not well-quasi-ordered with respect to the induced subgraph relation. Because of this, we can show show a stronger result for the first case.

Theorem 3 The class of $\left(\overline{2 P_{1}+P_{2}}, P_{2}+P_{4}, P_{6}\right)$-free graphs is not well-quasi-ordered by the induced subgraph relation.

Proof Let $n \geq 2$ be an integer and consider a cycle $C_{4 n}$, say $x_{1}-x_{2}-\cdots-x_{4 n}-x_{1}$. We partition the vertices of this cycle into three sets as follows:

$$
\begin{aligned}
& X=\left\{x_{i} \mid i \equiv 0 \operatorname{or} 2 \bmod 4\right\}, \\
& Y=\left\{x_{i} \mid i \equiv 1 \bmod 4\right\}, \\
& Z=\left\{x_{i} \mid i \equiv 3 \bmod 4\right\} .
\end{aligned}
$$

Let $G_{4 n}$ be the graph obtained from $C_{4 n}$ by connecting every vertex of $Y$ to every vertex of $Z$ (see also Fig. 2). Atminas and Lozin showed that the resulting graphs are $\left(\overline{2 P_{1}+P_{2}}, P_{6}\right)$ free and form an infinite antichain with respect to the induced subgraph relation [1].

It remains to prove that $G_{4 n}$ is $\left(P_{2}+P_{4}\right)$-free. We argue as in the proof of [11, Theorem 1 (iv)]. For contradiction, suppose that $G_{4 n}$ contains an induced subgraph $I$ isomorphic to $P_{2}+P_{4}$. Let $I_{1}$ and $I_{2}$ be the connected components of $I$ isomorphic to $P_{2}$ and $P_{4}$, respectively. Since $G_{4 n}[Y \cup Z]$ is complete bipartite, $I_{2}$ must contain at least one vertex of $X$. Since the two neighbours of any vertex of $X$ are adjacent, any vertex of $X$ in $I_{2}$ must be an end-vertex of $I_{2}$. Then, as $Y$ and $Z$ are independent sets, $I_{2}$ contains a vertex of both $Y$ and $Z$. As $I_{1}$ can contain at most one vertex of $X$ (because $X$ is an independent set), $I_{1}$ contains a vertex $u \in Y \cup Z$. However, $G_{4 n}[Y \cup Z]$ is complete bipartite and $I_{2}$ contains a vertex of both $Y$ and $Z$. Hence, $u$ has a neighbour in $I_{2}$, which is not possible. This completes the proof. 


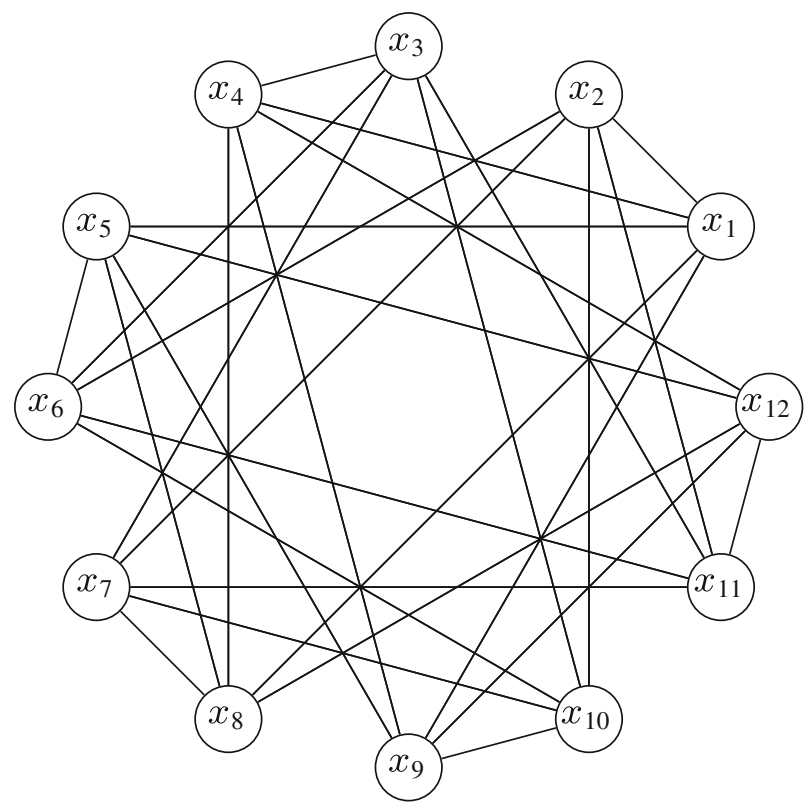

Fig. 3 The Graph $G_{4 n}$ from Theorem 4 when $n=3$

The graphs $G_{4 n}$ in the above proof are obtained from cycles in the same way that walls were transformed in [11] to show unboundedness of clique-width for $\left.\overline{\left(2 P_{1}+P_{2}\right.}, P_{2}+P_{4}\right)$ free graphs (The sets $A, B$ and $C$ in [11] correspond to the sets $Y, X$ and $Z$, respectively, in the proof above). In fact the construction in [11] is also $P_{6}$-free by the same arguments as in [1], so we obtain the following:

Remark 1 The class of $\left(\overline{2 P_{1}+P_{2}}, P_{2}+P_{4}, P_{6}\right)$-free graphs has unbounded clique-width.

For our second class, we need a new construction.

Theorem 4 The class of $\left(\overline{P_{1}+P_{4}}, P_{1}+2 P_{2}\right)$-free graphs is not well-quasi-ordered by the induced subgraph relation.

Proof Let $n \geq 3$ be an integer. Consider a cycle $C_{4 n}$, say $x_{1}-x_{2}-\cdots-x_{4 n}-x_{1}$. We partition the vertices of $C_{4 n}$ as follows:

$$
\begin{aligned}
& X=\left\{x_{i} \mid i \equiv 0 \text { or } 1 \bmod 4\right\}, \\
& Y=\left\{x_{i} \mid i \equiv 2 \text { or } 3 \bmod 4\right\} .
\end{aligned}
$$

Next we apply a complementation to each of $X$ and $Y$, so that in the resulting graph $X$ and $Y$ each induce a clique on $2 n$ vertices with a perfect matching removed. Let $G_{4 n}$ be the resulting graph (see also Fig. 3).

Suppose, for contradiction that $G_{4 n}$ contains an induced $P_{1}+2 P_{2}$. Without loss of generality, the set $X$ must contain three of the vertices $v_{1}, v_{2}, v_{3}$ of the $P_{1}+2 P_{2}$. Since every component of $P_{1}+2 P_{2}$ contains at most two vertices, without loss of generality we may assume that $v_{1}$ is non-adjacent to both $v_{2}$ and $v_{3}$. However, every vertex 
of $G_{4 n}[X]$ has exactly one non-neighbour in $X$. This contradiction shows that $G_{4 n}$ is indeed $\left(P_{1}+2 P_{2}\right)$-free.

Every vertex in $X$ has exactly one neighbour in $Y$ and vice versa. This means that any $K_{3}$ in $G_{4 n}$ must lie entirely in $G_{4 n}[X]$ or $G_{4 n}[Y]$. Since $G_{4 n}[X]$ or $G_{4 n}[Y]$ are both complements of perfect matchings and every vertex of $\overline{P_{1}+P_{4}}$ lies in one of three induced $K_{3}$ 's, which are pairwise non-disjoint, it follows that $G_{4 n}$ is $\overline{P_{1}+P_{4}}$-free.

It remains to show that the graphs $G_{4 n}$ form an infinite antichain with respect to the induced subgraph relation. Since $n \geq 3$, every vertex in $X$ (resp. $Y$ ) has at least two neighbours in $X$ (resp. $Y$ ) that are pairwise adjacent. Therefore, given $x_{1}$, we can determine which vertices lie in $X$ and which lie in $Y$. Every vertex in $X$ (resp. $Y$ ) has a unique neighbour in $Y$ (resp. $X$ ) and a unique non-neighbour in $X$ (resp. $Y$ ). Therefore, by specifying which vertex in $G_{4 n}$ is $x_{1}$, we uniquely determine $x_{2}, \ldots, x_{4 n}$. Suppose $G_{4 n}$ an induced subgraph of $G_{4 m}$ for some $m \geq 3$. Then $n \leq m$ due to the number of vertices. By symmetry, we may assume that the induced copy of $G_{4 n}$ in $G_{4 m}$ has vertex $x_{1}$ of $G_{4 n}$ in the position of vertex $x_{1}$ in $G_{4 m}$. Then the induced copy of $G_{4 n}$ must have vertices $x_{2}, \ldots, x_{4 n}$ in the same position as $x_{2}, \ldots, x_{4 n}$ in $G_{4 m}$, respectively. Now $x_{1}$ and $x_{4 n}$ are non-adjacent in $G_{4 n}$. If $n<m$ then $x_{1}$ and $x_{4 n}$ are adjacent in $G_{4 m}$, a contradiction. We conclude that if $G_{4 n}$ is an induced subgraph of $G_{4 m}$ then $n=m$. In other words $\left\{G_{4 n} \mid n \geq 3\right\}$ is an infinite antichain with respect to the induced subgraph relation.

\section{State of the Art and Future Work}

In this section we summarise what is currently known about well-quasi-orderability and boundedness of clique-width, taking into account the results proved in this paper. We also give a number of directions for future work.

Given four graphs $H_{1}, H_{2}, H_{3}, H_{4}$, the class of $\left(H_{1}, H_{2}\right)$-free graphs and the class of $\left(\mathrm{H}_{3}, \mathrm{H}_{4}\right)$-free graphs are equivalent if the unordered pair $\mathrm{H}_{3}, \mathrm{H}_{4}$ can be obtained from the unordered pair $H_{1}, H_{2}$ by some combination of the operations (i) complementing both graphs in the pair and (ii) if one of the graphs in the pair is $K_{3}$, replacing it with $\overline{P_{1}+P_{3}}$ or vice versa. If two classes are equivalent, then one of them is well-quasi-ordered with respect to the induced subgraph relation if and only if the other one is [21]. Similarly, if two classes are equivalent, then one of them has bounded clique-width if and only if the other one does [14]. We use this terminology in the remainder of this section.

\subsection{Well-Quasi-Ordering}

Atminas and Lozin [1] proved that the class of $\left(K_{3}, P_{6}\right)$-free graphs is well-quasi-ordered by the induced subgraph relation, while the class of $\left(\overline{2 P_{1}+P_{2}}, P_{6}\right)$-free graphs is not. In a recent paper [13] we proved that the classes of $\left(\overline{P_{1}+P_{3}}, P_{2}+P_{4}\right)$-free graphs and $\left(\overline{P_{1}+P_{3}}, P_{1}+P_{5}\right)$-free graphs are well-quasi-ordered by the labelled induced subgraph relation. Updating the classification in [21] with these four results and the three results proved in this paper (Theorems 2-4) leads to the following classification:

Theorem 5 Let $\mathcal{G}$ be a class of graphs defined by two forbidden induced subgraphs. Then:

1. $\mathcal{G}$ is well-quasi-ordered by the labelled induced subgraph relation if it is equivalent to a class of $\left(H_{1}, H_{2}\right)$-free graphs such that one of the following holds:

(i) $\mathrm{H}_{1}$ or $\mathrm{H}_{2} \subseteq_{i} P_{4}$; 
(ii) $H_{1}=s P_{1}$ and $H_{2}=K_{t}$ for some $s, t$;

(iii) $H_{1} \subseteq i \overline{P_{1}+P_{3}}$ and $H_{2} \subseteq_{i} P_{2}+P_{4}, P_{1}+P_{5}$ or $P_{6}$.

(iv) $H_{1} \subseteq_{i} \overline{2 P_{1}+P_{2}}$ and $H_{2} \subseteq_{i} P_{2}+P_{3}$ or $P_{5}$.

2. $\mathcal{G}$ is not well-quasi-ordered by the induced subgraph relation if it is equivalent to a class of $\left(H_{1}, H_{2}\right)$-free graphs such that one of the following holds:

(i) neither $H_{1}$ nor $H_{2}$ is a linear forest (disjoint union of paths);

(ii) $H_{1} \supseteq_{i} K_{3}$ and $H_{2} \supseteq_{i} 3 P_{1}+P_{2}, 3 P_{2}$ or $2 P_{3}$;

(iii) $H_{1} \supseteq_{i} \overline{2 P_{2}}$ and $H_{2} \supseteq_{i} 4 P_{1}$ or $2 P_{2}$;

(iv) $H_{1} \supseteq_{i} \overline{2 P_{1}+P_{2}}$ and $H_{2} \supseteq_{i} 4 P_{1}, P_{2}+P_{4}$ or $P_{6}$;

(v) $H_{1} \supseteq_{i} \overline{P_{1}+P_{4}}$ and $H_{2} \supseteq_{i} P_{1}+2 P_{2}$.

Note that in Theorem 5 every class that is well-quasi-ordered with respect to the induced subgraph relation is also well-quasi-ordered with respect to the labelled induced subgraph relation (see [1,21] and Theorem 2). This agrees with a conjecture of Atminas and Lozin [1] stating that these concepts coincide for hereditary classes $X$ precisely when $X$ is defined by a finite collection of forbidden induced subgraphs. Theorem 5 leaves us with six open cases.

Open Problem 1 Is the class of $\left(H_{1}, H_{2}\right)$-free graphs well-quasi-ordered by the induced subgraph relation when:

(i) $H_{1}=\overline{2 P_{1}+P_{2}}$ and $H_{2} \in\left\{P_{1}+2 P_{2}, P_{1}+P_{4}\right\}$;

(ii) $H_{1}=\overline{P_{1}+P_{4}}$ and $H_{2} \in\left\{P_{1}+P_{4}, 2 P_{2}, P_{2}+P_{3}, P_{5}\right\}$.

In relation to Open Problem 1, we mention that the infinite antichain for $\left(\overline{P_{1}+P_{4}}, P_{1}+2 P_{2}\right)$-free graphs was initially found by a computer search. This computer search also showed that similar antichains do not exist for any of the remaining six open cases. As such, constructing antichains for these cases is likely to be a challenging problem and this suggests that many of these classes may in fact be well-quasi-ordered. Some of these remaining classes have been shown to have bounded clique-width [3-5, 10]. We believe that some of the structural characterizations for proving these results may be useful for showing well-quasi-orderability.

\subsection{Clique-Width}

In the aforementioned paper [13] we also proved that the class of $\left(\overline{P_{1}+P_{3}}, P_{2}+P_{4}\right)$ free graphs has bounded clique-width. The following theorem, obtained after updating the theorem from [10] with the above result, describes exactly for which pairs $\left(H_{1}, H_{2}\right)$ the (un)boundedness of the clique-width of $\left(H_{1}, H_{2}\right)$-free graphs has been determined.

Theorem 6 Let $\mathcal{G}$ be a class of graphs defined by two forbidden induced subgraphs. Then:

1. $\mathcal{G}$ has bounded clique-width if it is equivalent to a class of $\left(H_{1}, H_{2}\right)$-free graphs such that one of the following holds:

(i) $\mathrm{H}_{1}$ or $\mathrm{H}_{2} \subseteq_{i} P_{4}$;

(ii) $H_{1}=s P_{1}$ and $H_{2}=K_{t}$ for some $s, t$;

(iii) $H_{1} \subseteq_{i} P_{1}+P_{3}$ and $\overline{H_{2}} \subseteq_{i} K_{1,3}+3 P_{1}, K_{1,3}+P_{2}, P_{1}+P_{2}+P_{3}, P_{1}+P_{5}$, $P_{1}+S_{1,1,2}, P_{2}+P_{4}, P_{6}, S_{1,1,3}$ or $S_{1,2,2}$;

(iv) $H_{1} \subseteq i 2 P_{1}+P_{2}$ and $\overline{H_{2}} \subseteq_{i} P_{1}+2 P_{2}, 2 P_{1}+P_{3}, 3 P_{1}+P_{2}$ or $P_{2}+P_{3}$; 
(v) $H_{1} \subseteq_{i} P_{1}+P_{4}$ and $\overline{H_{2}} \subseteq_{i} P_{1}+P_{4}$ or $P_{5}$;

(vi) $H_{1} \subseteq_{i} 4 P_{1}$ and $\overline{H_{2}} \subseteq_{i} 2 P_{1}+P_{3}$;

(vii) $H_{1}, \overline{H_{2}} \subseteq i K_{1,3}$.

2. $\mathcal{G}$ has unbounded clique-width if it is equivalent to a class of $\left(H_{1}, H_{2}\right)$-free graphs such that one of the following holds:

(i) $H_{1} \notin \mathcal{S}$ and $H_{2} \notin \mathcal{S}$;

(ii) $\overline{H_{1}} \notin \mathcal{S}$ and $\overline{H_{2}} \notin \mathcal{S}$;

(iii) $H_{1} \supseteq_{i} K_{1,3}$ or $2 P_{2}$ and $\overline{H_{2}} \supseteq_{i} 4 P_{1}$ or $2 P_{2}$;

(iv) $H_{1} \supseteq_{i} 2 P_{1}+P_{2}$ and $\overline{H_{2}} \supseteq_{i} K_{1,3}, 5 P_{1}, P_{2}+P_{4}$ or $P_{6}$;

(v) $H_{1} \supseteq_{i} 3 P_{1}$ and $\overline{H_{2}} \supseteq_{i} 2 P_{1}+2 P_{2}, 2 P_{1}+P_{4}, 4 P_{1}+P_{2}, 3 P_{2}$ or $2 P_{3}$;

(vi) $H_{1} \supseteq_{i} 4 P_{1}$ and $\overline{H_{2}} \supseteq_{i} P_{1}+P_{4}$ or $3 P_{1}+P_{2}$.

This leaves us with the following seven non-equivalent open cases.

Open Problem 2 Does the class of $\left(H_{1}, H_{2}\right)$-free graphs have bounded or unbounded clique-width when:

(i) $H_{1}=3 P_{1}$ and $\overline{H_{2}} \in\left\{P_{1}+S_{1,1,3}, S_{1,2,3}\right\}$;

(ii) $H_{1}=2 P_{1}+P_{2}$ and $\overline{H_{2}} \in\left\{P_{1}+P_{2}+P_{3}, P_{1}+P_{5}\right\}$;

(iii) $H_{1}=P_{1}+P_{4}$ and $\overline{H_{2}} \in\left\{P_{1}+2 P_{2}, P_{2}+P_{3}\right\}$ or

(iv) $H_{1}=\overline{H_{2}}=2 P_{1}+P_{3}$.

A potential direction for future research related to determining boundedness of cliquewidth is investigating linear clique-width for classes defined by two forbidden induced subgraphs. Indeed, it is not hard to show that $k$-uniform graphs have bounded linear cliquewidth. Again, we can use complementations and vertex deletions when dealing with this parameter.

\subsection{Well-Quasi-Ordering versus Clique-Width}

Recall that all bigenic classes known to be well-quasi-ordered by the induced subgraph relation are also known to have bounded clique-width. Hence, to verify Conjecture 1 for bigenic classes, we only need to check the six open cases mentioned in Open Problem 1. Note that Conjecture 1 is verified directly if a graph class has bounded clique-width. Brandstädt, Le and Mosca [4] proved that the class of $\left(\overline{P_{1}+P_{4}}, P_{1}+P_{4}\right)$-free graphs (and thus the class of $\left(\overline{2 P_{1}+P_{2}}, P_{1}+P_{4}\right)$-free graphs) has bounded clique-width. Using results from [3], the same authors proved in [5] that the class of $\left(\overline{P_{1}+P_{4}}, P_{5}\right)$-free graphs (and thus the class of $\left(\overline{P_{1}+P_{4}}, 2 P_{2}\right)$-free graphs) has bounded clique-width. Dabrowski, Dross and Paulusma [10] showed that the class of $\left(\overline{2 P_{1}+P_{2}}, P_{1}+2 P_{2}\right)$-free graphs has bounded clique-width. Hence, there is only one bigenic class of graphs left for which Conjecture 1 needs to be verified.

Open Problem 3 Is Conjecture 1 true for the class of $\left(H_{1}, H_{2}\right)$-free graphs when $H_{1}=$ $\overline{P_{1}+P_{4}}$ and $H_{2}=P_{2}+P_{3}$ ?

As can be seen from Open Problems 1 and 2, we known neither whether the class of $\left(\overline{P_{1}+P_{4}}, P_{2}+P_{3}\right)$-free graphs is well-quasi-ordered by the induced subgraph relation nor whether its clique-width is bounded. 
Open Access This article is distributed under the terms of the Creative Commons Attribution 4.0 International License (http://creativecommons.org/licenses/by/4.0/), which permits unrestricted use, distribution, and reproduction in any medium, provided you give appropriate credit to the original author(s) and the source, provide a link to the Creative Commons license, and indicate if changes were made.

\section{Appendix A: Proofs of the Claims in Lemma 8 from [11, Lemma 10]}

Claim 1. $G\left[V_{i} \cup X\right]$ is a matching.

Indeed, if some vertex $x$ in $V_{i}$ (respectively $X$ ) is adjacent to two vertices $y_{1}, y_{2}$ in $X$ (respectively $V_{i}$ ), then $G\left[v_{i+2}, v_{i+3}, y_{1}, x, y_{2}\right]$ is a $P_{2}+P_{3}$.

Claim 2. If $V_{i}$ and $V_{j}$ are opposite, then $G\left[V_{i} \cup V_{j}\right]$ is a matching.

Suppose for contradiction that $x \in V_{1}$ is adjacent to two vertices $y, y^{\prime} \in V_{3}$. Then $G\left[v_{2}, x, y, y^{\prime}\right]$ would be a $\overline{2 P_{1}+P_{2}}$, a contradiction.

Claim 3. If $V_{i}$ and $V_{j}$ are consecutive, then $G\left[V_{i} \cup V_{j}\right]$ is a co-matching.

Suppose for contradiction that $x \in V_{1}$ is non-adjacent to two vertices $y, y^{\prime} \in V_{2}$. Then $G\left[x, v_{5}, y, v_{3}, y^{\prime}\right]$ is a $P_{2}+P_{3}$, a contradiction.

Claim 4. If $V_{i}$ is large, then $X$ is anti-complete to $V_{i-2} \cup V_{i+2} \int$.

Suppose for contradiction that $V_{3}$ is large and $x \in X$ has a neighbour $y \in V_{1}$. Then since $V_{3}$ is large and both $G\left[X \cup V_{3}\right]$ and $G\left[V_{1} \cup V_{3}\right]$ are matchings, there must be a vertex $z \in V_{3}$ that is non-adjacent to both $x$ and $y$. Then $G\left[x, y, v_{3}, v_{4}, z\right]$ is a $P_{2}+P_{3}$, a contradiction.

Claim 5. If $V_{i}$ is large, then $V_{i-1}$ is anti-complete to $V_{i+1}$.

Suppose for contradiction that $V_{2}$ is large and $x \in V_{1}$ has a neighbour $y \in V_{3}$. Since $V_{2}$ is large and each vertex in $V_{1} \cup V_{3}$ has at most one non-neighbour in $V_{2}$, there must be a vertex $z \in V_{2}$ that is adjacent to both $x$ and $y$. Now $G\left[x, y, v_{2}, z\right]$ is a $\overline{2 P_{1}+P_{2}}$, a contradiction.

Claim 6. If $V_{i-1}, V_{i}, V_{i+1}$ are large, then $V_{i}$ is complete to $V_{i-1} \cup V_{i+1}$.

Suppose for contradiction that $V_{1}, V_{2}, V_{3}$ are large and some vertex $x \in V_{1}$ is non-adjacent to a vertex $y \in V_{2}$. Since $V_{3}$ is large and $G\left[V_{2} \cup V_{3}\right]$ is a co-matching, there must be two vertices $z, z^{\prime} \in V_{3}$, adjacent to $y$. By the previous claim, since $V_{2}$ is large, $z, z^{\prime}$ must be non-adjacent to $x$. Therefore $G\left[x, v_{5}, z, y, z^{\prime}\right]$ is a $P_{2}+P_{3}$, which is a contradiction.

\section{Appendix B: Proofs of the Claims in Lemma 9 from [11, Lemma 11]}

Claim 1. $V_{i}$ is independent for $i \in\{1,2\}$.

If $x, y \in V_{i}$ were adjacent then $G\left[x, y, v_{i+1}, v_{i+3}\right]$ would be a $\overline{2 P_{1}+P_{2}}$.

Claim 2. $W_{i}$ is independent for $i \in\{1,2,3,4\}$.

If $x, y \in W_{i}$ were adjacent then $G\left[x, y, v_{i+1}, v_{i+2}, v_{i+3}\right]$ would be a $P_{2}+P_{3}$.

Claim 3. $X$ is independent.

If $x, y \in X$ were adjacent then $G\left[x, y, v_{1}, v_{2}, v_{3}\right]$ would be a $P_{2}+P_{3}$.

Claim 4. $W_{i}$ is anti-complete to $X$ for $i \in\{1,2,3,4\}$.

If $x \in X$ were adjacent to $y \in W_{i}$ then $G\left[x, y, v_{i+1}, v_{i+2}, v_{i+3}\right]$ would be a $P_{2}+P_{3}$.

Claim 5. For $i \in\{1,2\}$ either $W_{i}$ or $W_{i+2}$ is empty. Therefore, we may assume by symmetry that $W_{3}=\emptyset$ and $W_{4}=\emptyset$.

To show this, first suppose that vertices $x \in W_{1}$ and $y \in W_{3}$ are adjacent. Then $G\left[v_{1}, v_{2}, v_{3}, y, x\right]$ is a $C_{5}$, which is a contradiction. Therefore, $W_{1}$ is anti-complete to $W_{3}$. If both $W_{1}$ and $W_{3}$ are non-empty then by our earlier assumption they must each contain at least two vertices. Suppose that $x \in W_{1}$ and $y, z \in W_{3}$. In this case $G\left[x, v_{1}, y, v_{3}, z\right]$ is a $P_{2}+P_{3}$, a contradiction. We conclude that at least one of $W_{1}$ and $W_{3}$ must be empty. Without loss of generality, we assume that $W_{3}$ is empty. Similarly, we assume that $W_{4}$ is empty. 


\section{References}

1. Atminas, A., Lozin, V.V.: Labelled induced subgraphs and well-quasi-ordering. Order 32(3), 313-328 (2015)

2. Bodlaender, H.L.: A linear-time algorithm for finding tree-decompositions of small treewidth. SIAM J. Comput. 25(6), 1305-1317 (1996)

3. Brandstädt, A., Kratsch, D.: On the structure of $\left(P_{5}\right.$, gem $)$-free graphs. Discret. Appl. Math. 145(2), 155-166 (2005)

4. Brandstädt, A., Le, H.-O., Mosca, R.: Gem- and co-gem-free graphs have bounded clique-width. Int. J. Found. Comput. Sci. 15(1), 163-185 (2004)

5. Brandstädt, A., Le, H.-O., Mosca, R.: Chordal co-gem-free and ( $P_{5}$,gem)-free graphs have bounded clique-width. Discret. Appl. Math. 145(2), 232-241 (2005)

6. Courcelle, B.: The monadic second-order logic of graphs III:, tree-decompositions, minor and complexity issues. Informatique Théorique et Applications 26(3), 257-286 (1992)

7. Courcelle, B.: Clique-width and edge contraction. Inf. Process. Lett. 114(1-2), 42-44 (2014)

8. Courcelle, B., Makowsky, J.A., Rotics, U.: Linear time solvable optimization problems on graphs of bounded clique-width. Theory of Computing Systems 33(2), 125-150 (2000)

9. Courcelle, B., Olariu, S.: Upper bounds to the clique width of graphs. Discret. Appl. Math. 101(1-3), 77-114 (2000)

10. Dabrowski, K.K., Dross, F., Paulusma, D.: Colouring diamond-free graphs. Proc. SWAT, 2016 LIPIcs 53, 16:1-16:14 (2016)

11. Dabrowski, K.K., Huang, S., Paulusma, D.: Bounding clique-width via perfect graphs. Journal of Computer and System Sciences, (in press)

12. Dabrowski, K.K., Lozin, V.V., Paulusma, D.: Well-quasi-ordering versus clique-width: New results on bigenic classes. Proc. IWOCA 2016, LNCS 9843, 253-265 (2016)

13. Dabrowski, K.K., Lozin, V.V., Paulusma, D.: Clique-Width and Well-Quasi Ordering of Triangle-Free Graph Classes. Proceedings WG 2017 LNCS, (to appear)

14. Dabrowski, K.K., Paulusma, D.: Clique-width of graph classes defined by two forbidden induced subgraphs. Comput. J. 59(5), 650-666 (2016)

15. Daligault, J., Rao, M., Thomassé, S.: Well-quasi-order of relabel functions. Order 27(3), 301-315 (2010)

16. Damaschke, P.: Induced subgraphs and well-quasi-ordering. Journal of Graph Theory 14(4), 427-435 (1990)

17. Espelage, W., Gurski, F., Wanke, E.: How to solve NP-hard graph problems on clique-width bounded graphs in polynomial time. Proc. WG 2001, LNCS 2204, 117-128 (2001)

18. Finkel, A., Schnoebelen, P.: Well-structured transition systems everywhere! Theor. Comput. Sci. 256(12), 63-92 (2001)

19. Higman, G.: Ordering by divisibility in abstract algebras. Proc. Lond. Math. Soc. s3-2(1), 326-336 (1952)

20. Kobler, D., Rotics, U.: Edge dominating set and colorings on graphs with fixed clique-width. Discret. Appl. Math. 126(2-3), 197-221 (2003)

21. Korpelainen, N., Lozin, V.V.: Two forbidden induced subgraphs and well-quasi-ordering. Discret. Math. 311(16), 1813-1822 (2011)

22. Kruskal, J.B.: The theory of well-quasi-ordering: A frequently discovered concept. Journal of Combinatorial Theory Series A 13(3), 297-305 (1972)

23. Lozin, V.V., Razgon, I., Zamaraev, V.: Well-quasi-ordering does not imply bounded clique-width. Proc. WG 2015, LNCS 9224, 351-359 (2016)

24. Oum, S.-I., Seymour, P.D.: Approximating clique-width and branch-width. Journal of Combinatorial Theory Series B 96(4), 514-528 (2006)

25. Rao, M.: MSOL partitioning problems on graphs of bounded treewidth and clique-width. Theor. Comput. Sci. 377(1-3), 260-267 (2007)

26. Robertson, N., Seymour, P.D.: Graph minors. IV. Tree-width and well-quasi-ordering. Journal of Combinatorial Theory Series B 48(2), 227-254 (1990)

27. Robertson, N., Seymour, P.D.: Graph minors. XX. Wagner's conjecture. Journal of Combinatorial Theory Series B 92(2), 325-357 (2004) 